\section{Dexamethasone-related adrenal insufficiency in patients with solid brain tumours}

\author{
Authors: Helen Currie, Muzzammil Ali Supervisor: Dr. Andrew Toogood (Consultant \\ Endocrinologist)
}

University Hospitals Nis

Birmingham

NHS Foundation Trus

\section{RATIONALE}

Primary brain tumours, although fairly uncommon (8 per 100,000 per year) comprise the 8 th most common cancer of people of working age (16-65) and the fifth most common cause of death from cancer in under $65 \mathrm{~s}$. The mainstay of treatment for primary brain tumours is surgical excision and radiotherapy, and in two thirds of patients, steroids (dexamethasone) is used to reduce cerebral oedema and to help relieve the symptoms of raised intracranial pressure such as headache and vomiting. Long term use of high dose exogenous steroids in such a manner can cause suppression of the hypothalamic-pituitary-adrenal (HPA) axis impacting both on corticotrophin-releasing hormone $(\mathrm{CRH})$ and adrenocorticotrophic hormone (ACTH) as demonstrated below.

With prolonged suppression of the HPA axis, adrenal glands eventually atrophy and can take months to years to recover some degree of functioning. Undiagnosed

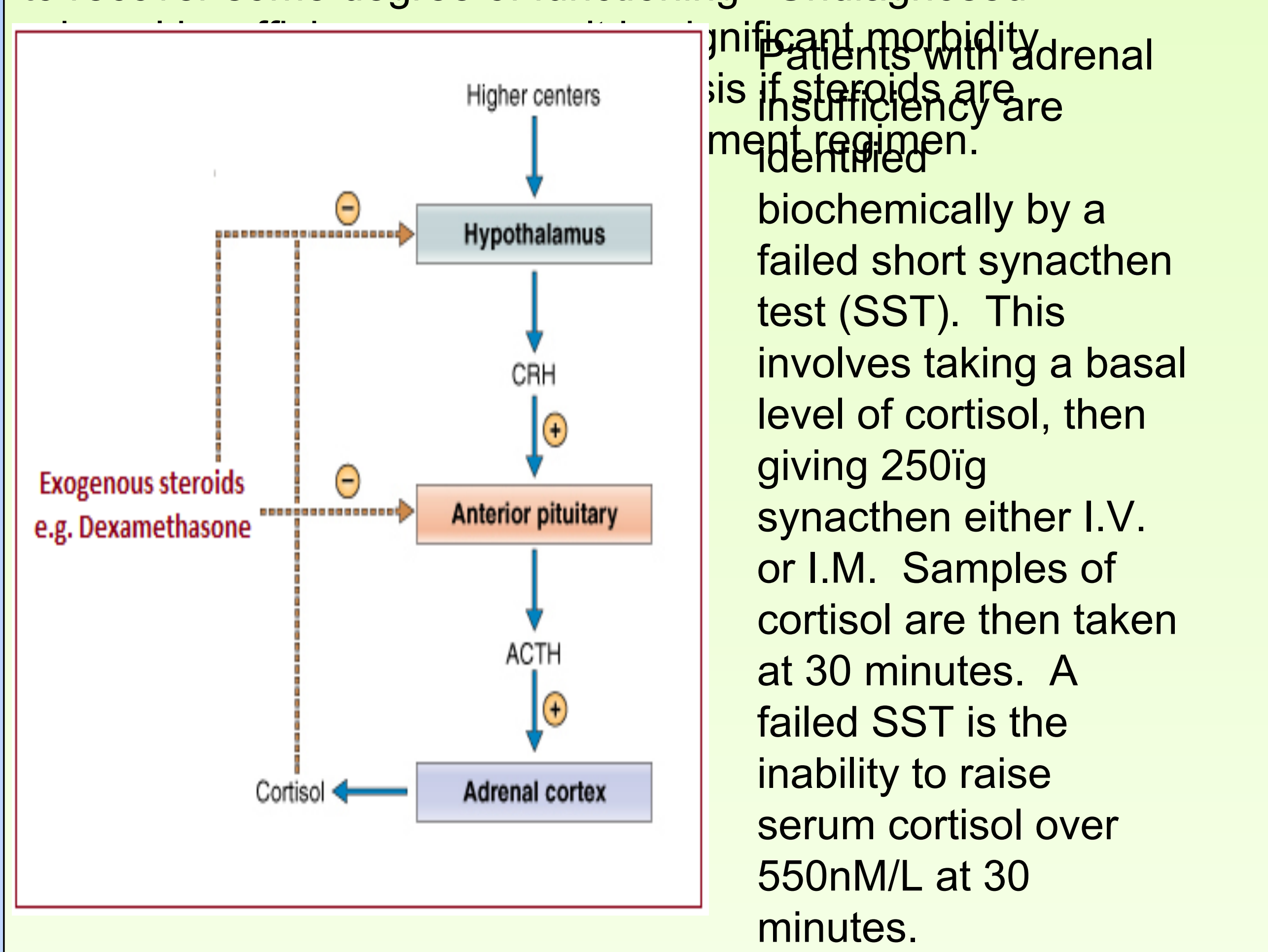
failed.

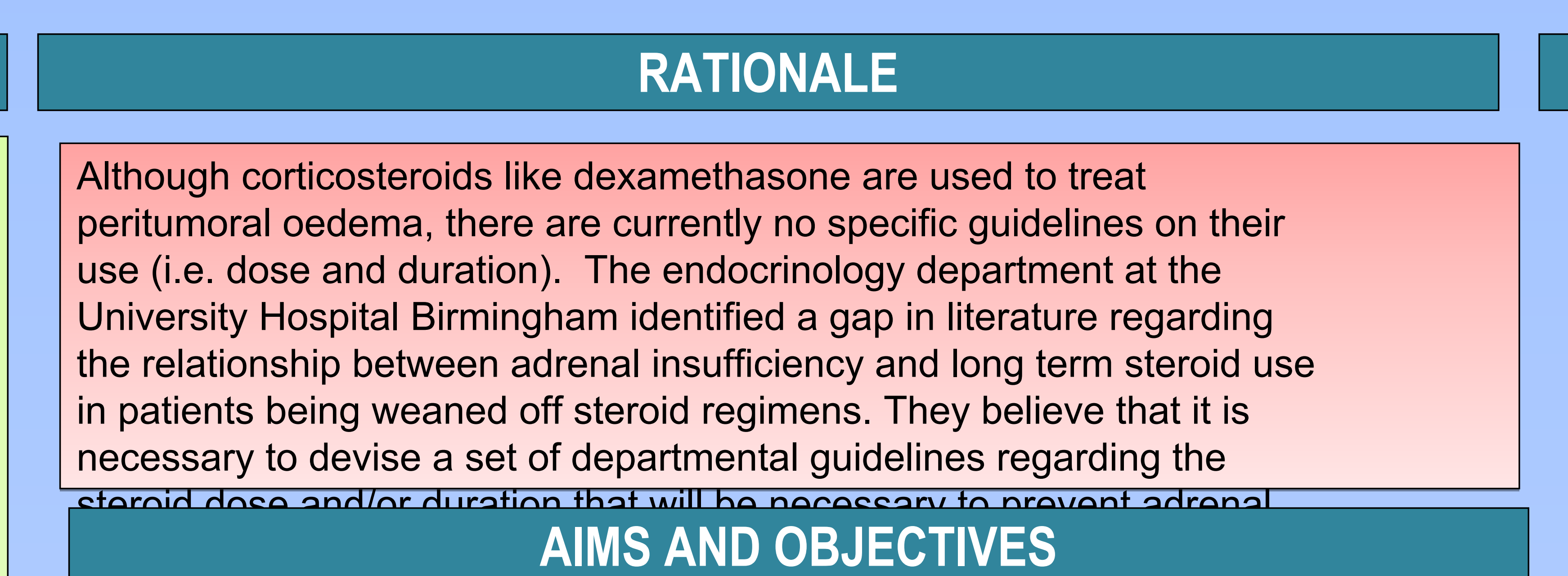

1. To determine the median dose, duration and dexamethasone exposure in both groups: those who passed their SST and those who

2. To determine whether there is a significance difference in the baseline cortisol, 30 minute cortisol, median dose, median duration and median total steroid exposure between the 2 groups

3. To propose a dose and/or duration of steroid exposure that may

\section{orevent adrenal insufficiencv.}



Information on demographics, diagnosis, baseline and 30 minute cortisol, steroid type, dose and duration of treatment was obtained for each patient. Every time the dose was altered, this was recorded to give an end result of area under the curve i.e. total steroid exposure. Statistical analysis was then carried out to see if there was any significant difference between the pass and fail groups.

\section{RESULTS}

\section{Patient Characteristics}

Biochemistry lists identified 1,164 patients who had SSTs between October 2010 and October 2012 Informatics used specific clinic codes to identify patients who had attended brain tumour endocrinology clinics. After review on clinical portal, a total of 78 patients were obtained that fit the selection criteria of this study population of 78 , 53 patients passed their SST and 25 failed.

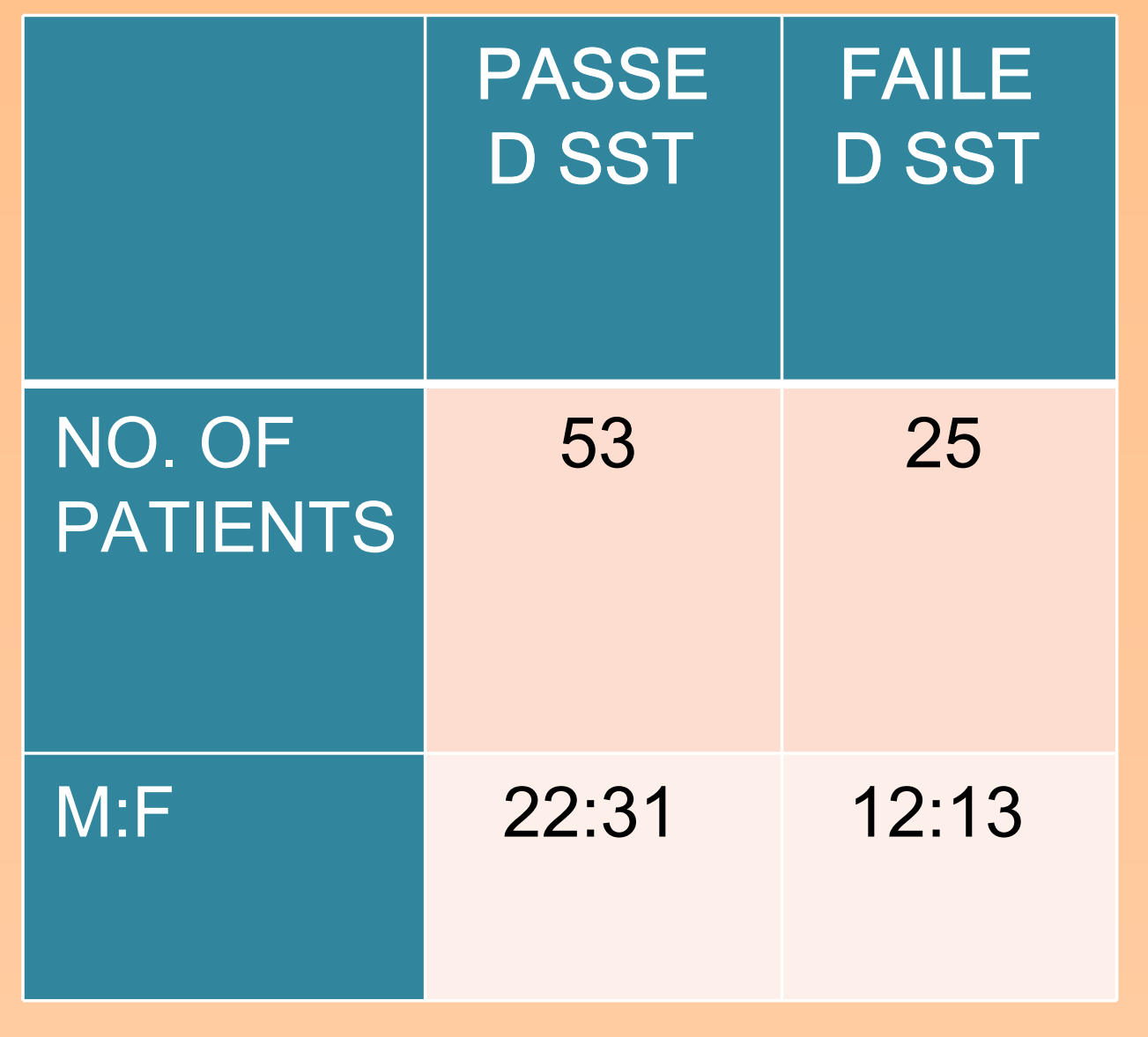

Solid Brain Tumour Diagnosis

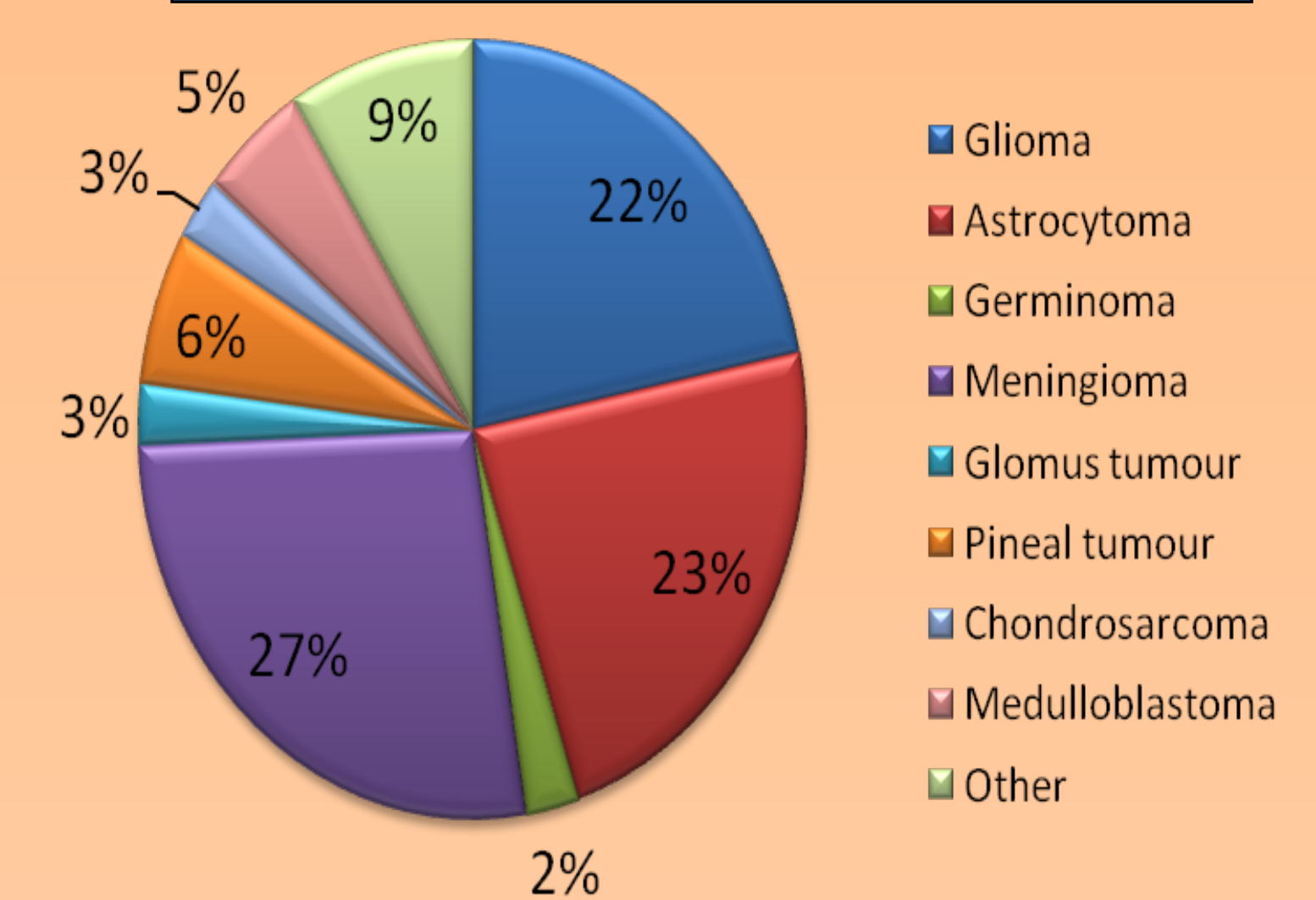

The pie chart above shows the confirmed diagnosis of all patients in both the pass and fail groups
Comparison of those who passed SST and those who failed SST

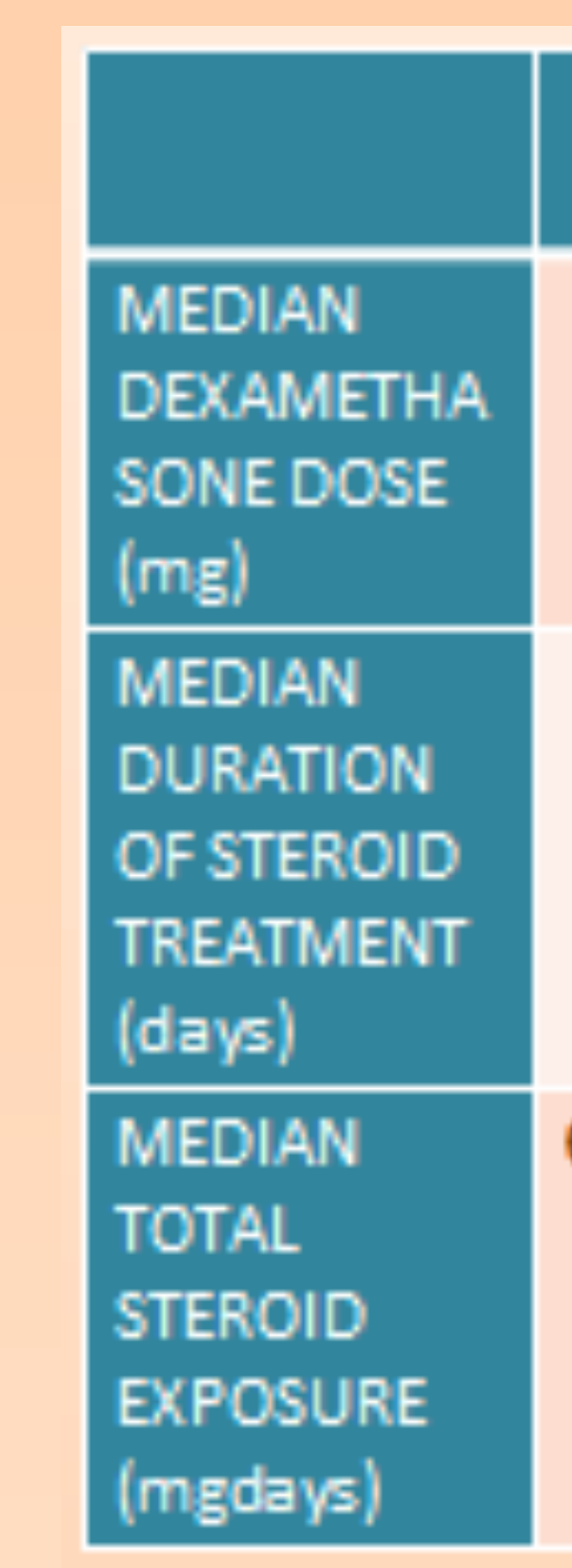

\begin{tabular}{|c|c|}
\hline $\begin{array}{l}\text { PASSED } \\
\text { SST }\end{array}$ & FAILED SST \\
\hline $\begin{array}{l}0.00 \mathrm{mg} \\
\text { (IQR= } \\
2.3 \mathrm{mg})\end{array}$ & $\begin{array}{c}3.30 \mathrm{mg} \\
\text { (IQR= } \\
2.70 \mathrm{mg})\end{array}$ \\
\hline $\begin{array}{c}0 \text { days } \\
(\text { IQR }=56 \\
\text { days) }\end{array}$ & $\begin{array}{c}360 \text { days } \\
(\text { IQR }=390 \\
\text { days })\end{array}$ \\
\hline $\begin{array}{c}0.00 \mathrm{mgday} \\
5 \\
(\text { IQR }=88 \\
\text { mgdays })\end{array}$ & $\begin{array}{c}928 \\
\text { mgdays } \\
(\text { IQR= } 1920 \\
\text { mgdays })\end{array}$ \\
\hline
\end{tabular}
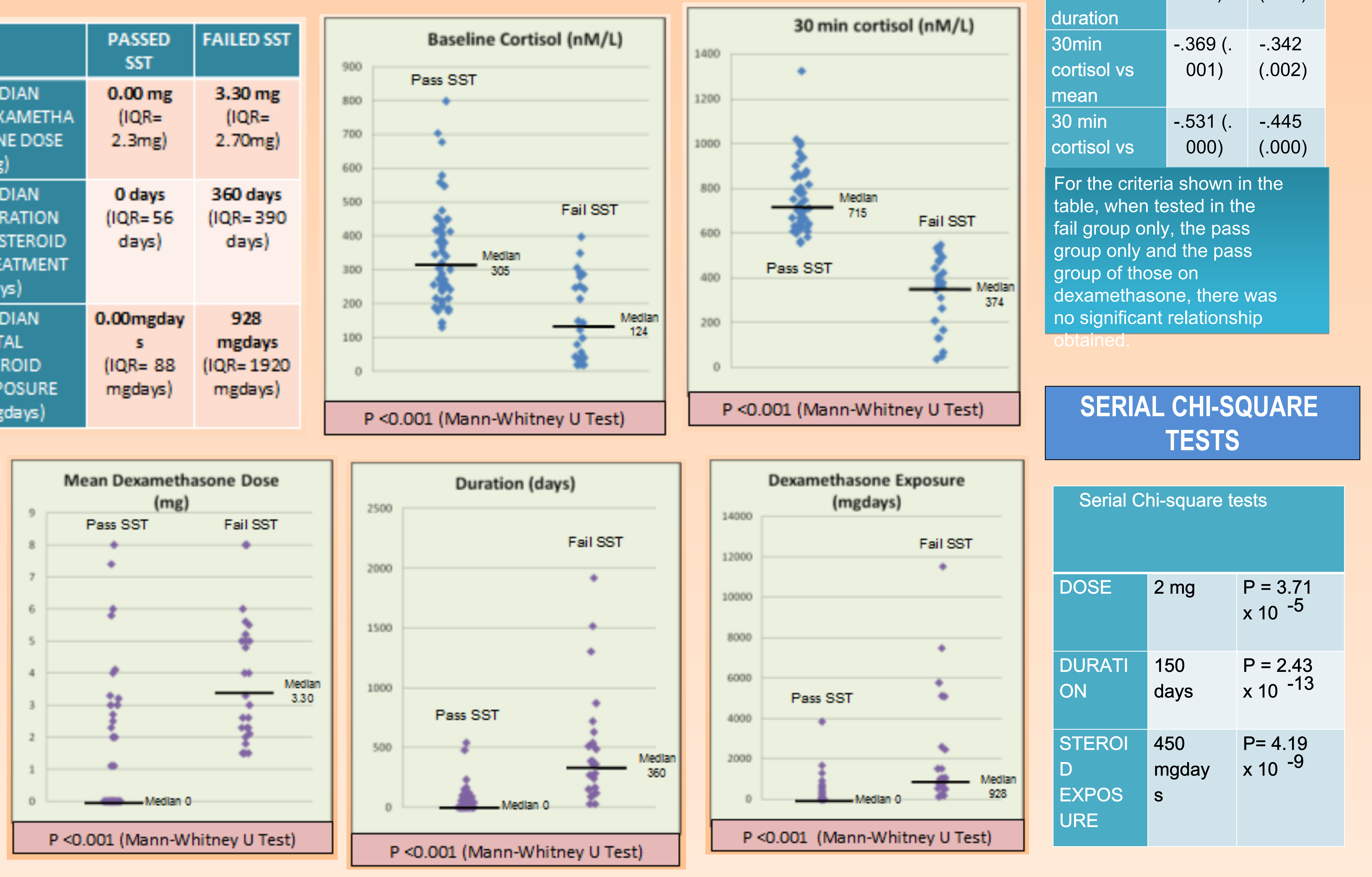

Correlation tests:

\section{DISCUSSION \\ The primary aim was to determine the relationship between adrenal insufficiency and long term steroid exposure. \\ The pass and fail groups were compared itially with respect to 5 variables:
$\tilde{n}$ Baseline cortisol
$\tilde{\eta} 30$ minute cortisol
$\tilde{n}$ Mean dose
$\tilde{\eta}$ Mean duration \\ $\tilde{\eta}$ Total steroid exposure.}

A Mann-Whitney $U$ test compared whether there was a significant difference between the pass and fail groups for the aforementioned variables. Scatterplots show, in all categories, the null hypothesis was rejected; there was a significant difference between those who passed their SST and those who failed.

Spearman 's and Pearson 's correlation tests were done on all the data to determine whether any correlations existed. Tests were performed first on the pass vs. fail group as a whole. There was a significant negative correlation between baseline cortisol and both steroid duration and exposure. However, no significant correlation existed between baseline cortisol and dose. A noted between 30 minute cortisol and all the variables: duration, dose and exposure. This showed that exposure and duration were more influential factors for failing SST, rather than dose. Spearman and Pearson correlation tests were then conducted on 2 additional groups: the fail group only and the pass group only. None of the variables showed a significant correlation with baseline cortisol or with 30 minute cortisol.

In the pass group $62 \%$ of the subjects (33 out of 53 patients) had a mean dose of $0 \mathrm{mg}$ dexamethasone i.e. no exposure. Therefore, Spearman 's and Pearson 's tests were also carried out on those patients in the pass group who had been exposed to steroids only (the 33 patients who had not were excluded from this correlation). This was done to determine whether the lack of steroid exposure in the pass group had an impact on the results. No significant difference was found however between those on dexamethasone and those who were not exposed to steroids in the pass group for all the aforementioned variables. Additionally, after removing those who were not on steroids, a comparison of pass and fail groups showed that there was still a significant difference in duration and exposure. This strengthens the point that the groups are indeed different, independent of the lack of steroid exposure in the pass group.

The findings suggest a threshold point for failing an SST; once a person fails an SST at a particular point, it does not matter how much

additional steroid exposure there is, they

have already developed adrenal

\section{RECOMMENDATIONS}

It has been found that patients with solid brain tumours are more likely to develop adrenal insufficiency when on dexamethasone:

Dose: $>2 \mathrm{mg}$, Duration: $>150$ days , Exposure $>450$ mgdays.

This demonstrates that patients on dexamethasone for $>5$ months or an

exposure $>450 \mathrm{mgdays}$ will almost certainly

need hydrocortisone. These patients do not need an SST, they are at high risk of adrenal

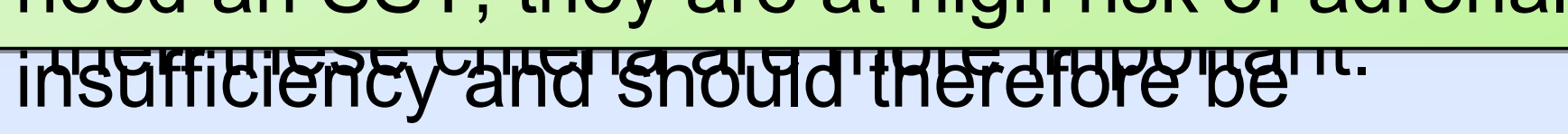

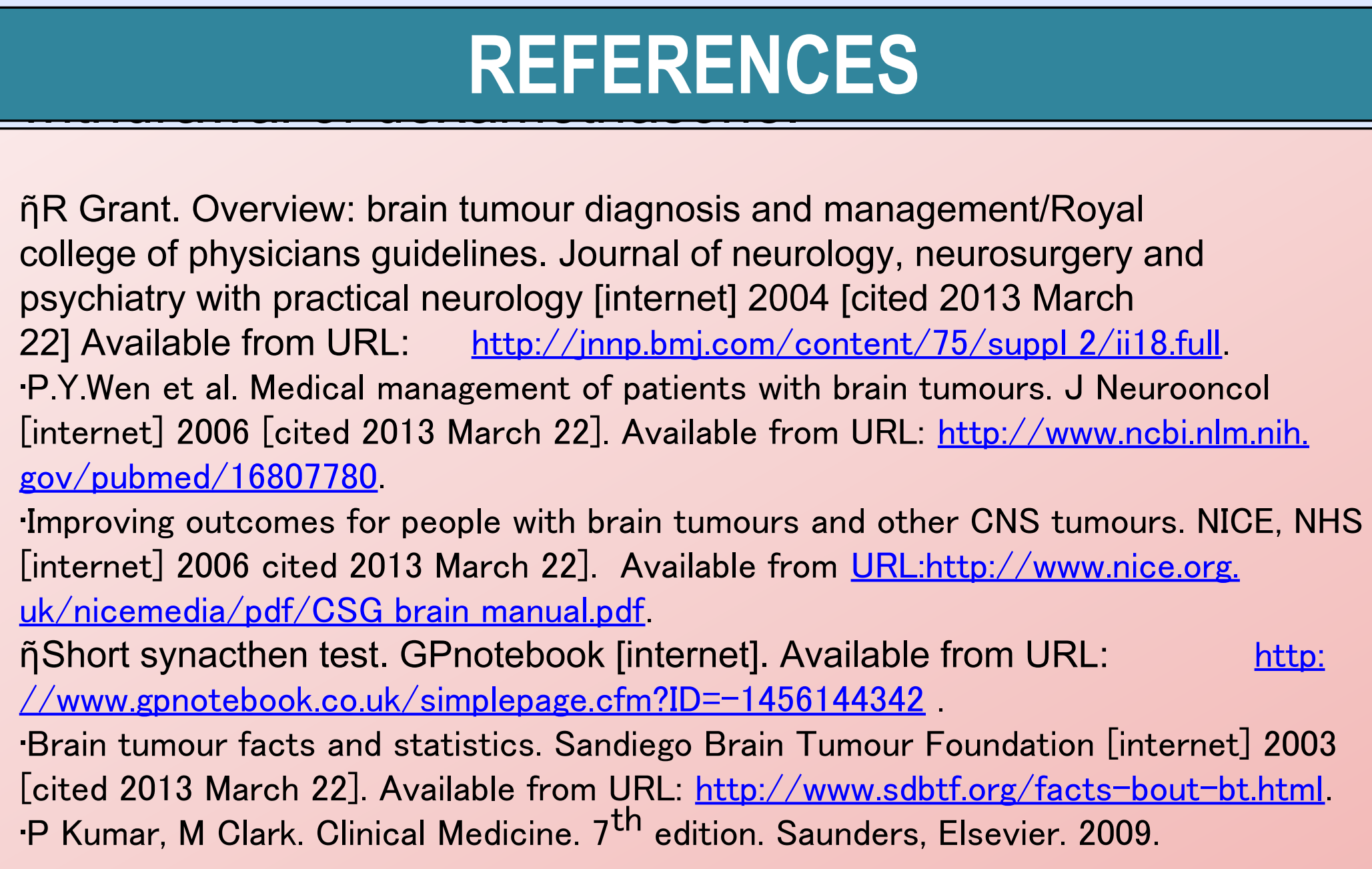

